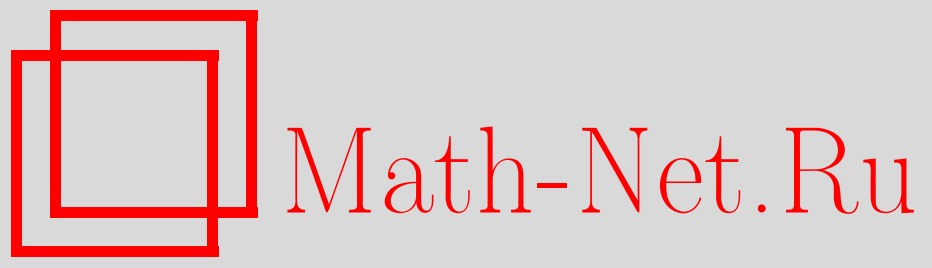

В. П. Демичев, Оптимальная оценка ковариации индикаторных функций от ассоциированных случайных величин, Теория вероятн. и ее примен., 2013, том 58, выпуск 4, 804-812

DOI: https://doi.org/10.4213/tvp4543

Использование Общероссийского математического портала Math-Net.Ru подразумевает, что вы прочитали и согласны с пользовательским соглашением http://www.mathnet.ru/rus/agreement

Параметры загрузки:

IP : 54.237 .59 .107

26 апреля 2023 г., 14:19:28

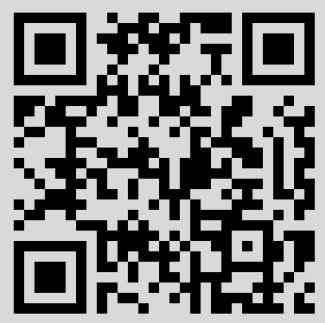


6. Новичкая O.С., Яцало Е. Б. Экстремальное поведение рекуррентных случайных последовательностей. - Вестн. Моск. ун-та. Матем. мех., 2008, № 5, с. 6-10.

Поступила в редакцию 2.II.2013

(c) 2013 г

\title{
ОПТИМАЛЬНАЯ ОЦЕНКА КОВАРИАЦИИ ИНДИКАТОРНЫХ ФУНКЦИЙ ОТ АССОЦИИРОВАННЫХ СЛУЧАЙНЫХ ВЕЛИЧИН ${ }^{1)}$
}

ДЕМИЧЕВ В. П.*

\begin{abstract}
Получена оптимальная оценка для ковариации индикаторных функций от положительно или отрицательно ассоциированных случайных величин, улучшающая неравенство, которое установили Багай и Пракаса Рао. Рассматриваются приложения доказанного результата к исследованию предельных свойств эмпирических функций распределения и объемов экскурсионных множеств случайных полей.
\end{abstract}

Ключевые слова и фразы: ассоциированность, ковариация, индикаторные функции.

Введение. Важный класс случайных полей образуют системы случайных величин, обладающих свойством ассоциированности и его модификациями (см., например, [1]). При доказательстве предельных теорем для таких полей существенную роль играет условие конечной восприимчивости, предложенное Ч. Ньюменом [2]. Для стационарных в широком смысле полей, заданных на решетке $\mathbf{Z}^{d}$, это означает суммируемость ковариационной функции. Во многих приложениях проверка этого условия осложнена невозможностью явного вычисления последней. Однако часто можно представить элементы исследуемого случайного поля в виде некоторых функций от элементов другого ассоциированного поля, ковариационная функция которого известна. Индикаторные функции представляют собой важный частный случай, возникающий, например, при исследовании эмпирических распределений и экскурсионных множеств случайных полей. Соответственно, интерес представляет задача оценки ковариации индикаторных функций от случайных величин через ковариацию исходных величин.

Оценка такого рода появилась в работах [3] и [4] в предположении существования у рассматриваемых ассоциированных случайных величин ограниченных плотностей. В [5] было получено обобщение этой оценки на случай, когда плотности, вообще говоря, не ограничены, но принадлежат пространству $L_{p}(\mathbf{R}), p>1$. В данной работе мы улучшаем оценку из [3] и [4], а также доказываем, что наша оценка оптимальна. Заметим, что в [6] (теоремы 1 и 3) получены более точные оценки, однако на характер зависимости рассматриваемых величин налагаются весьма жесткие ограничения.

1. Формулировка основных результатов. Напомним несколько основных понятий, которые понадобятся нам, чтобы сформулировать результаты работы.

Согласно [7] семейство случайных величин $\xi=\left\{\xi_{t}, t \in T\right\}$ называется ассоциированным (пишут $\xi \in \mathrm{A}$ ), если для любых конечных подмножеств $I=\left\{t_{1}, \ldots, t_{n}\right\} \subset T, J=\left\{s_{1}, \ldots, s_{m}\right\} \subset T$ и ограниченных покоординатно неубывающих функций $F: \mathbf{R}^{n} \rightarrow \mathbf{R}$ и $G: \mathbf{R}^{m} \rightarrow \mathbf{R}$ выполнено неравенство

$$
\operatorname{cov}\left(F\left(\xi_{t_{1}}, \ldots, \xi_{t_{n}}\right), G\left(\xi_{s_{1}}, \ldots, \xi_{s_{m}}\right)\right) \geqslant 0 .
$$

* Московский государственный университет им. М. В. Ломоносова, механикоматематический факультет, кафедра теории вероятностей, Россия, Москва, ГСП-1, Ленинские горы 1; e-mail: vadim.demichev@gmail.com

1) Работа выполнена при частичной поддержке гранта РФФИ № 10-01-00397-а. 
В случае, когда (1.1) имеет место при дополнительном предположении $I \cap J=\varnothing$, говорят, что семейство $\xi$ положительно (слабо) ассоциированно $(\xi \in \mathrm{PA})$, см. [8]. Если по-прежнему $I \cap J=\varnothing$, но знак неравенства (1.1) заменить на противоположный (см. [9]), то получится определение отрицательной ассоцированности $(\xi \in \mathrm{NA})$.

Очевидно, что ассоциированность семейства случайных величин влечет положительную ассоциированность. В то же время обратное утверждение неверно даже для двухэлементных семейств (см. [7]).

Будем также говорить, что случайный вектор $\xi=\left(\xi_{1}, \ldots, \xi_{n}\right), n \in \mathbf{N}$, ассоциирован (соответственно, положительно ассоциирован или отрицательно ассоциирован) и писать $\xi \in \mathrm{A}(\xi \in \mathrm{PA}$ или $\xi \in \mathrm{NA})$, если множество случайных величин, составленное из его компонент, обладает этим свойством. Запись $\xi \in \mathrm{L}_{2}$ означает, что $\xi_{i} \in \mathrm{L}_{2}(\Omega)$, $i=1, \ldots, n$.

Пусть $X, Y$ - квадратично интегрируемые случайные величины с функциями распределения (ф.р.) $F_{X}, F_{Y}$ и ограниченными плотностями $p_{X}, p_{Y}$ соответственно. В [3] показано, что если $(X, Y) \in \mathrm{A}$, то для любого $T>0$

$$
\sup _{x, y \in \mathbf{R}} \operatorname{cov}(\mathbf{I}\{X>x\}, \mathbf{I}\{Y>y\}) \leqslant C_{1}\left(T^{2} \operatorname{cov}(X, Y)+\frac{1}{T}\left(\left\|p_{X}\right\|_{\infty}+\left\|p_{Y}\right\|_{\infty}\right)\right),
$$

где $\mathbf{I}\{\cdot\}$ обозначает индикатор события. Здесь и далее $C_{1}, C_{2}, \ldots, c_{1}, c_{2}, \ldots-$ некоторые положительные константы. Минимизируя последнее выражение по $T>0$, получаем

$$
\sup _{x, y \in \mathbf{R}} \operatorname{cov}(\mathbf{I}\{X>x\}, \mathbf{I}\{Y>y\}) \leqslant C_{2}\left(\left(\left\|p_{X}\right\|_{\infty}+\left\|p_{Y}\right\|_{\infty}\right)^{2} \operatorname{cov}(X, Y)\right)^{1 / 3} .
$$

Записывая эту оценку для случайных величин $k_{1} X, k_{2} Y$ и минимизируя правую часть по $k_{1}, k_{2}>0$, имеем

$$
\sup _{x, y \in \mathbf{R}} \operatorname{cov}(\mathbf{I}\{X>x\}, \mathbf{I}\{Y>y\}) \leqslant C_{3}\left(\left\|p_{X}\right\|_{\infty}\left\|p_{Y}\right\|_{\infty} \operatorname{cov}(X, Y)\right)^{1 / 3} .
$$

Для упрощения записи будем использовать обозначения $x_{+}=\max \{x, 0\}, x_{-}=$ $\max \{-x, 0\}, x \vee y=\max \{x, y\}, x \wedge y=\min \{x, y\}, x, y \in \mathbf{R}$. Введем функцию $f$, которая монотонно и непрерывно отображает отрезок $[0,1]$ на себя, следующей формулой:

$$
f(0)=0 ; \quad f(x)=\frac{x^{2}}{\ln (e / x)}, \quad x \in(0,1] .
$$

В данной работе мы улучшаем оценку (1.2). А именно, верна следующая теорема.

Теорема 1.1. Если квадратично интегрируемый случайный вектор $(X, Y)$ положительно или отрицательно ассочиирован, то имеет место неравенство

$$
\sup _{x, y \in \mathbf{R}}|\operatorname{cov}(\mathbf{I}\{X>x\}, \mathbf{I}\{Y>y\})| \leqslant C_{4} f^{\mathrm{inv}}\left(\left\|p_{X}\right\|_{\infty}\left\|p_{Y}\right\|_{\infty}|\operatorname{cov}(X, Y)| \wedge 1\right),
$$

где $f^{\mathrm{inv}}$ - обратная функция $\kappa f$.

Оптимальность этой оценки устанавливает следующая теорема.

Теорема 1.2. Для любого $r \in(0,1]$ найдется случайньий вектор $(X, Y) \in \mathrm{PA} \cap \mathrm{L}_{2}$ maкой, ито $\operatorname{cov}(X, Y) \in(0, r],\left\|p_{X}\right\|_{\infty}=\left\|p_{Y}\right\|_{\infty}=1, u$

$$
\sup _{x, y \in \mathbf{R}} \operatorname{cov}(\mathbf{I}\{X>x\}, \mathbf{I}\{Y>x\}) \geqslant c_{1} f^{\text {inv }}(\operatorname{cov}(X, Y)) .
$$

Следствие 1.1. Для любого $0<\delta<1 / 2$ найдется такое $C=C(\delta)>0$, ито

$$
\sup _{x, y \in \mathbf{R}}|\operatorname{cov}(\mathbf{I}\{X>x\}, \mathbf{I}\{Y>y\})| \leqslant C\left(\left\|p_{X}\right\|_{\infty}\left\|p_{Y}\right\|_{\infty}|\operatorname{cov}(X, Y)|\right)^{\delta},
$$

если $(X, Y) \in \mathrm{PA} \cap \mathrm{L}_{2}$ или $(X, Y) \in \mathrm{NA} \cap \mathrm{L}_{2}$. В то же время оченку вида (1.4) нельзя получить при $\delta=1 / 2$. 
2. Доказательства. Введем функцию

$$
\begin{aligned}
H(x, y) & =\operatorname{cov}(\mathbf{I}\{X>x\}, \mathbf{I}\{Y>y\}) \\
& =F_{X, Y}(x, y)-F_{X}(x) F_{Y}(y), \quad x, y \in \mathbf{R},
\end{aligned}
$$

где $F_{X, Y}$ - функция распределения $(X, Y)$. Отметим, что если $(X, Y) \in \mathrm{PA}$ или $(X, Y) \in \mathrm{NA}$, то $H$ соответственно неотрицательна или неположительна во всей области определения. Также известно (см., например, [1, приложение, теорема 1.2]), что $|\operatorname{cov}(X, Y)|=\|H\|_{1}$.

Так как и левая и правая части соотношения (1.3) инвариантны относительно домножения $X$ и $Y$ на положительные числа, утверждение теоремы 1.1 эквивалентно выполнению неравенства

$$
\|H\|_{1} \geqslant c_{2} f\left(\|H\|_{\infty}\right)
$$

для всех положительно или отрицательно ассоциированных случайных величин $X, Y \in \mathrm{L}_{2}$ таких, что

$$
\left\|p_{X}\right\|_{\infty}=\left\|p_{Y}\right\|_{\infty}=1 .
$$

В свою очередь утверждение теоремы 1.2 эквивалентно существованию для каждого $r \in(0,1]$ такого случайного вектора $(X, Y) \in \mathrm{PA} \cap \mathrm{L}_{2}$ с $\operatorname{cov}(X, Y) \in(0, r]$ и $\left\|p_{X}\right\|_{\infty}=$ $\left\|p_{Y}\right\|_{\infty}=1$, что

$$
\|H\|_{1} \leqslant C_{5} f\left(\|H\|_{\infty}\right) .
$$

В предположениях $(X, Y) \in \mathrm{PA} \cap \mathrm{L}_{2}$ и $(2.2)$ легко проверяются следующие свойства функции $H$ :

(A1) $0 \leqslant H(x, y)<1, x, y \in \mathbf{R}$;

(A2) для любых $x, y \in \mathbf{R}, \Delta x, \Delta y>0$ выполнено неравенство

$$
H(x, y)+H(x+\Delta x, y+\Delta y)-H(x+\Delta x, y)-H(x, y+\Delta y) \geqslant-\Delta x \Delta y
$$

(A3) $\operatorname{Lip}_{1}(H) \vee \operatorname{Lip}_{2}(H) \leqslant 1$, где $\operatorname{Lip}_{1}(H), \operatorname{Lip}_{2}(H)$ - липшицевы константы $H$ по первой и второй переменной соответственно.

Если $(X, Y) \in \mathrm{NA} \cap \mathrm{L}_{2}$, то свойствами (A1)-(А3) обладает функция $G(x, y)=$ $-H(-x, y), x, y \in \mathbf{R}$. Поэтому теорема 1.1 будет доказана, если мы установим следующий результат.

Лемма 2.1. Для ююбой функиии $H: \mathbf{R}^{2} \rightarrow \mathbf{R}$, обладающей свойствами (A1)(А3), выполнено неравенство (2.1).

Теорема 1.2 является следствием следующих двух утверждений.

Лемма 2.2. Для каждого $r \in(0,1]$ найдется такая функиия $H: \mathbf{R}^{2} \rightarrow \mathbf{R}$, обладающая свойствами (A1) $u(\mathrm{~A} 2)$, что $\operatorname{Lip}_{1}(H) \vee \operatorname{Lip}_{2}(H) \leqslant 1 / 4, \operatorname{supp}(H) \subset$ $[-1 / 4,1 / 4]^{2},\|H\|_{1} \in(0, r]$, и выполнено $(2.3)$.

Лемма 2.3. Для каждой функции $H$, удовлетворяющей условиям из формулировки леммы 2.2, найдется такой случайный вектор $(X, Y) \in \mathrm{PA} \cap \mathrm{L}_{2}$, ито выполнено свойство (2.2), и $H(x, y)=\operatorname{cov}(\mathbf{I}\{X>x\}, \mathbf{I}\{Y>y\}), x, y \in \mathbf{R}$.

Перед тем как перейти непосредственно к доказательству лемм 2.1-2.3, мы установим еще несколько вспомогательных утверждений.

Введем класс $\mathscr{G}$, состоящий из финитных непрерывных действительных функций $g$, заданных на $(-\infty, 0]$. Определим оператор $S$ формулой

$$
S[g](x)=g(x)+\left(\inf _{s \leqslant x} g(s)\right)_{-}, \quad g \in \mathscr{G}, \quad x \in(-\infty, 0] .
$$

Предложение 2.1. Для любых $g, g_{1}, g_{2} \in \mathscr{G}$ и допустимых значений аргументов справедливы следующие утверждения:

(B1) $S[g](x) \geqslant g(x)_{+}$;

(В2) если $x_{1}<x_{2}$, то выполнено неравенство $S[g]\left(x_{2}\right)-S[g]\left(x_{1}\right) \geqslant g\left(x_{2}\right)-g\left(x_{1}\right)$;

(В3) если непрерьвная функиия $h(x) \geqslant 0$ такова, что для любых $x_{1}<x_{2}$ выполнено неравенство $h\left(x_{2}\right)-h\left(x_{1}\right) \geqslant g\left(x_{2}\right)-g\left(x_{1}\right)$, mо $h(x) \geqslant S[g](x)$ при всех $x$;

(B4) если для любых $x_{1}<x_{2}$ выполнено неравенство $g_{1}\left(x_{2}\right)-g_{1}\left(x_{1}\right) \leqslant g_{2}\left(x_{2}\right)-$ $g_{2}\left(x_{1}\right)$, mо $S\left[g_{1}\right](x) \leqslant S\left[g_{2}\right](x)$ при всех $x$. 
Д о к а з а т е л ь с т в о. Утверждения (B1) и (В2) вытекают непосредственно из определения $S$. Докажем (B3). Пусть $S[g](x)>0$ для некоторого $x \leqslant 0$. Положим $x_{0}=\sup \{s<x: S[g](s)=0\}$. При $t \in\left(x_{0}, x\right]$ имеем

$$
0<S[g](t)=g(t)+\left(\inf _{s \leqslant t} g(s)\right)_{-},
$$

следовательно, $\left(\inf _{s \leqslant x} g(s)\right)_{-}=\left(\inf _{s \leqslant x_{0}} g(s)\right)_{-}$. Поэтому

$$
S[g](x)=S[g](x)-S[g]\left(x_{0}\right)=g(x)-g\left(x_{0}\right) \leqslant h(x)-h\left(x_{0}\right) \leqslant h(x) .
$$

Докажем (B4). В силу (В2) для любых $x_{1}<x_{2}$

$$
S\left[g_{2}\right]\left(x_{2}\right)-S\left[g_{2}\right]\left(x_{1}\right) \geqslant g_{2}\left(x_{2}\right)-g_{2}\left(x_{1}\right) \geqslant g_{1}\left(x_{2}\right)-g_{1}\left(x_{1}\right),
$$

следовательно, (В4) вытекает из (В3). Предложение 2.1 доказано.

Для $w>0$ и $y \geqslant 0$ определим оператор $T_{y}^{w}$ формулой

$$
T_{y}^{w}[g](x)=g(x)-y(x+w)_{+}, \quad g \in \mathscr{G}, \quad x \in(-\infty, 0] .
$$

Предложение 2.2. При любых $w>0, y \geqslant 0 u g \in \mathscr{G}$ имеет место равенство

$$
S\left[T_{y}^{w}[g]\right](x)=S\left[T_{y}^{w}[S[g]]\right](x), \quad x \in(-\infty, 0] .
$$

Д о к а з а т е л ь с т в о. Возьмем произвольные $x_{1}<x_{2} \leqslant 0$. Имеем

$$
\begin{gathered}
\left(T_{y}^{w}[S[g]]\left(x_{2}\right)-T_{y}^{w}[S[g]]\left(x_{1}\right)\right)-\left(T_{y}^{w}[g]\left(x_{2}\right)-T_{y}^{w}[g]\left(x_{1}\right)\right) \\
=\left(S[g]\left(x_{2}\right)-S[g]\left(x_{1}\right)\right)-\left(g\left(x_{2}\right)-g\left(x_{1}\right)\right) .
\end{gathered}
$$

В силу (В2) последнее выражение неотрицательно. Значит, по свойству (В4) выполнено неравенство $S\left[T_{y}^{w}[g]\right](x) \leqslant S\left[T_{y}^{w}[S[g]]\right](x), x \leqslant 0$. Остается проверить, что $S\left[T_{y}^{w}[g]\right](x) \geqslant S\left[T_{y}^{w}[S[g]]\right](x), x \leqslant 0$. В силу (В3) достаточно показать, что для всех $x_{1}<x_{2} \leqslant 0$

$$
S\left[T_{y}^{w}[g]\right]\left(x_{2}\right)-S\left[T_{y}^{w}[g]\right]\left(x_{1}\right) \geqslant T_{y}^{w}[S[g]]\left(x_{2}\right)-T_{y}^{w}[S[g]]\left(x_{1}\right) .
$$

Для любого $x \leqslant 0$ справедливы равенства

$$
\begin{gathered}
S\left[T_{y}^{w}[g]\right](x)=g(x)-y(x+w)_{+}+\left(\inf _{s \leqslant x}\left(g(s)-y(s+w)_{+}\right)\right)_{-}, \\
T_{y}^{w}[S[g]](x)=g(x)-y(x+w)_{+}+\left(\inf _{s \leqslant x} g(s)\right)_{-}
\end{gathered}
$$

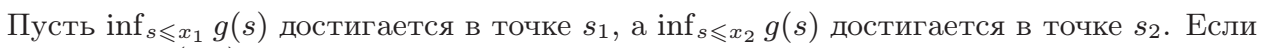
$s_{2} \leqslant x_{1}$, то (2.4), очевидно, выполнено. Рассмотрим случай $s_{2}>x_{1}$. Имеем

$$
\begin{aligned}
\left(S\left[T_{y}^{w}[g]\right]\left(x_{2}\right)-S\left[T_{y}^{w}[g]\right]\left(x_{1}\right)\right)-\left(T_{y}^{w}[S[g]]\left(x_{2}\right)-T_{y}^{w}[S[g]]\left(x_{1}\right)\right) \\
=\left(\inf _{s \leqslant x_{2}}\left(g(s)-y(s+w)_{+}\right)\right)_{-}-\left(\inf _{s \leqslant x_{1}}\left(g(s)-y(s+w)_{+}\right)\right) \\
\quad-\left(g\left(s_{2}\right)_{-}-g\left(s_{1}\right)_{-}\right) .
\end{aligned}
$$

Кроме того,

$$
\begin{aligned}
\left(\inf _{s \leqslant x_{1}}\left(g(s)-y(s+w)_{+}\right)\right)_{-} & \leqslant\left(g\left(s_{1}\right)-y\left(x_{1}+w\right)_{+}\right)_{-} \\
\left(\inf _{s \leqslant x_{2}}\left(g(s)-y(s+w)_{+}\right)\right)_{-} & \geqslant\left(g\left(s_{2}\right)-y\left(s_{2}+w\right)_{+}\right)_{-} \\
& \geqslant\left(g\left(s_{2}\right)-y\left(x_{1}+w\right)_{+}\right)_{-} .
\end{aligned}
$$

Поэтому

$$
\begin{aligned}
& \left(S\left[T_{y}^{w}[g]\right]\left(x_{2}\right)-S\left[T_{y}[g]\right]\left(x_{1}\right)\right)-\left(T_{y}^{w}[S[g]]\left(x_{2}\right)-T_{y}^{w}[S[g]]\left(x_{1}\right)\right) \\
& \quad \geqslant\left(g\left(s_{2}\right)-y\left(x_{1}+w\right)_{+}\right)_{-}-\left(g\left(s_{1}\right)-y\left(x_{1}+w\right)_{+}\right)_{-}-\left(g\left(s_{2}\right)_{-}-g\left(s_{1}\right)_{-}\right) \geqslant 0 .
\end{aligned}
$$


Предложение 2.2 доказано.

Д о к а з а т е л ь с т в о л е м м ы 2.1. Если $\|H\|_{\infty}=0$, то $\|H\|_{1}=0$, и искомая оценка тривиальна. Пусть $\|H\|_{\infty}>0$. Без ограничения общности предполагаем, что $\sup _{x, y \in \mathbf{R}} H(x, y)$ достигается в точке $(0,0): H(0,0)=\|H\|_{\infty}>0$. Отметим, что можно считать функцию $H$ финитной: $\operatorname{supp}(H) \subset[-1,1]^{2}$. Действительно, положим

$$
G(x, y)=\frac{1}{9} H(x, y) \varphi(x) \varphi(y), \quad x, y \in \mathbf{R},
$$

где $\varphi(x)=\exp \left\{-1 /\left(1-x^{2}\right)\right\} \mathbf{I}\{|x|<1\}$. Очевидно, что $\|G\|_{1} \leqslant\|H\|_{1}$, и в то же время $\|G\|_{\infty} \geqslant\|H\|_{\infty} / 9 e^{2}$. Кроме того, пользуясь оценкой $\left\|\varphi^{\prime}\right\|_{\infty} \leqslant 2$, нетрудно проверить, что $G$ обладает свойствами (А1)-(А3).

Введем функцию $U(x, y)=S\left[T_{y}^{1}[H(\cdot, 0)]\right](x), x \leqslant 0, y \geqslant 0$. По свойству (В4) оператора $S$ функция $U(x, y)$ не возрастает по $y$ при всех $x \leqslant 0$. Непосредственно из определений операторов $S$ и $T_{y}^{1}, y \geqslant 0$, вытекает вложение $\operatorname{supp}(U) \subset[-1,0] \times[0, \infty)$. В силу оценки $\operatorname{Lip}_{1}(H) \leqslant 1$ функция $T_{y}^{1}[H(\cdot, 0)]$ неположительна и не возрастает при $y \geqslant 1$, следовательно, $\operatorname{supp}(U) \subset[-1,0] \times[0,1]$. Так как $H$ обладает свойством (A2), для любых $-1 \leqslant x_{1}<x_{2} \leqslant 0$ и $y \geqslant 0$ выполнено неравенство

$$
\begin{aligned}
& H\left(x_{1}, 0\right)+H\left(x_{2}, y\right)-H\left(x_{2}, 0\right)-H\left(x_{1}, y\right) \geqslant-y\left(x_{2}-x_{1}\right) \\
& \quad \Leftrightarrow H\left(x_{2}, y\right)-H\left(x_{1}, y\right) \geqslant H\left(x_{2}, 0\right)-H\left(x_{1}, 0\right)-y\left(x_{2}-x_{1}\right) \\
& \quad=T_{y}^{1}[H(\cdot, 0)]\left(x_{2}\right)-T_{y}^{1}[H(\cdot, 0)]\left(x_{1}\right) .
\end{aligned}
$$

Поэтому по свойству (В3) $H(x, y) \geqslant U(x, y), x \leqslant 0, y \geqslant 0$. Таким образом, $\|H\|_{1} \geqslant\|U\|_{1}$. Кроме того, очевидно, что $U(0,0)=H(0,0)$. А значит, достаточно доказать оценку $\|U\|_{1} \geqslant c_{3} f(U(0,0))$.

Для $k \in \mathbf{Z}_{+}$введем $y_{k}=a k / 2$, где $a=U(0,0)$. Воспользовавшись предложением 2.2 , получаем, что для любых $k \in \mathbf{Z}_{+}$и $-1 \leqslant x \leqslant 0$

$$
\begin{aligned}
& U\left(0, y_{k+1}\right)-U\left(x, y_{k+1}\right)=S\left[T_{y_{k+1}}^{1}[H(\cdot, 0)]\right](0)-S\left[T_{y_{k+1}}^{1}[H(\cdot, 0)]\right](x) \\
& \quad=S\left[T_{y_{k+1}-y_{k}}^{1}\left[T_{y_{k}}^{1}[H(\cdot, 0)]\right]\right](0)-S\left[T_{y_{k+1}-y_{k}}^{1}\left[T_{y_{k}}^{1}[H(\cdot, 0)]\right]\right](x) \\
& \quad=S\left[T_{y_{k+1}-y_{k}}^{1}\left[S\left[T_{y_{k}}^{1}[H(\cdot, 0)]\right]\right]\right](0)-S\left[T_{y_{k+1}-y_{k}}^{1}\left[S\left[T_{y_{k}}^{1}[H(\cdot, 0)]\right]\right]\right](x) \\
& \quad=S\left[T_{y_{k+1}-y_{k}}^{1}\left[U\left(\cdot, y_{k}\right)\right]\right](0)-S\left[T_{y_{k+1}-y_{k}}^{1}\left[U\left(\cdot, y_{k}\right)\right]\right](x) .
\end{aligned}
$$

По свойству (B2) последнее выражение не меньше

$$
T_{y_{k+1}-y_{k}}^{1}\left[U\left(\cdot, y_{k}\right)\right](0)-T_{y_{k+1}-y_{k}}^{1}\left[U\left(\cdot, y_{k}\right)\right](x)=U\left(0, y_{k}\right)-U\left(x, y_{k}\right)+x\left(y_{k+1}-y_{k}\right) .
$$

Следовательно,

$$
U\left(x, y_{k}\right) \geqslant U\left(x, y_{k+1}\right)+U\left(0, y_{k}\right)-U\left(0, y_{k+1}\right)+x\left(y_{k+1}-y_{k}\right) .
$$

Так как $U\left(x, y_{k}\right) \geqslant U\left(x, y_{k+1}\right)$, то

$$
U\left(x, y_{k}\right) \geqslant U\left(x, y_{k+1}\right)+\left(U\left(0, y_{k}\right)-U\left(0, y_{k+1}\right)+x\left(y_{k+1}-y_{k}\right)\right)_{+} .
$$

Интегрируя последнее соотношение по $x \in[-1,0]$, получаем оценку

$$
\int_{-1}^{0} U\left(x, y_{k}\right) d x \geqslant \int_{-1}^{0} U\left(x, y_{k+1}\right) d x+\frac{\left(U\left(0, y_{k}\right)-U\left(0, y_{k+1}\right)\right)^{2}}{2\left(y_{k+1}-y_{k}\right)} .
$$

Здесь мы воспользовались неравенством $U\left(0, y_{k}\right)-U\left(0, y_{k+1}\right) \leqslant y_{k+1}-y_{k}$, которое вытекает из (2.5) при $x=-1$.

Положим $N=\lfloor 2 / a\rfloor$, где $\lfloor\cdot\rfloor$ — целая часть числа. Имеем

$$
\int_{-1}^{0} U\left(x, y_{k}\right) d x \geqslant \sum_{l=k}^{N} \frac{\left(U\left(0, y_{l}\right)-U\left(0, y_{l+1}\right)\right)^{2}}{2\left(y_{l+1}-y_{l}\right)}=\frac{1}{a} \sum_{l=k}^{N}\left(U\left(0, y_{l}\right)-U\left(0, y_{l+1}\right)\right)^{2} .
$$


Обозначая $d_{k}=U\left(0, y_{k}\right)-U\left(0, y_{k+1}\right), k=1, \ldots, N$, получаем

$$
\int_{0}^{\infty} \int_{-1}^{0} U(x, y) d x d y \geqslant \sum_{k=1}^{N}\left(y_{k}-y_{k-1}\right) \int_{-1}^{0} U\left(x, y_{k}\right) d x \geqslant \frac{1}{2} \sum_{k=1}^{N} k d_{k}^{2} .
$$

Поскольку $U(0, y)=0$ при $y \geqslant 1$, по свойству (B1)

$$
\sum_{k=1}^{N} d_{k}=U\left(0, y_{1}\right)-U\left(0, y_{N+1}\right)=U\left(0, y_{1}\right) \geqslant T_{y_{1}}^{1}[H(\cdot, 0)](0)=H(0,0)-y_{1}=\frac{a}{2} .
$$

Минимизируя (2.6) при условии $(2.7)$ по $d_{k} \geqslant 0, k=1, \ldots, N$, имеем

$$
\|U\|_{1} \geqslant \frac{a^{2}}{12 \ln N} \geqslant \frac{1}{12} f(a)
$$

Лемма 2.1 доказана.

Д о к а 3 а т е ль с т в о л е м м ы 2.2. Пусть $a=f^{-1}(r) \in(0,1], N=\lfloor 4 / a\rfloor$. Введем $d_{k}=a /(64 k \ln N), k \in \mathbf{N}$. Рассмотрим монотонно возрастающую функцию $g(x), x \leqslant 0$, вида

$$
g(x)= \begin{cases}0, & x \leqslant-\frac{N a}{16} \\ \sum_{l=k+1}^{N} d_{l}+\frac{16}{a}\left(x-\left(-\frac{k a}{16}\right)\right) d_{k}, & -\frac{k a}{16} \leqslant x \leqslant-\frac{(k-1) a}{16}, \quad k=1, \ldots, N .\end{cases}
$$

Имеем $1>g(0)=\sum_{k=1}^{N} d_{k} \geqslant a / 64, \operatorname{Lip}(g) \leqslant 1 / 4, \operatorname{supp}(g) \subset[-1 / 4,0]$. Положим

$$
H(x, y)=S\left[T_{y}^{1 / 4}[g]\right](x), \quad x \leqslant 0, \quad y \geqslant 0 .
$$

Рассмотрим $k_{y}=\left\lfloor(4 y \ln N)^{-1}\right\rfloor \wedge N, y>0$. Нетрудно показать, что функция $H(\cdot, y)$ равна нулю при $x \leqslant-k_{y} a / 16$, не убывает при $-k_{y} a / 16 \leqslant x \leqslant 0$, и если $-k_{y} a / 16 \leqslant x_{1} \leqslant x_{2} \leqslant 0$, то

$$
H\left(x_{2}, y\right)-H\left(x_{1}, y\right)=T_{y}^{1 / 4}[g]\left(x_{2}\right)-T_{y}^{1 / 4}[g]\left(x_{1}\right)=g\left(x_{2}\right)-g\left(x_{1}\right)-y\left(x_{2}-x_{1}\right) .
$$

Следовательно, для любых $x_{1} \leqslant x_{2} \leqslant 0,0 \leqslant y_{1} \leqslant y_{2}$ верна оценка

$$
H\left(x_{2}, y_{1}\right)-H\left(x_{1}, y_{1}\right) \geqslant H\left(x_{2}, y_{2}\right)-H\left(x_{1}, y_{2}\right) .
$$

Так как $k_{y}=0$ при $y \geqslant 1 / 4$, то $\operatorname{supp}(H) \subset[-1 / 4,0] \times[0,1 / 4]$.

Оценим $\operatorname{Lip}_{1}(H)$ и $\operatorname{Lip}_{2}(H)$. Возьмем любые $-1 / 4 \leqslant x_{1} \leqslant x_{2} \leqslant 0,0 \leqslant y_{1} \leqslant y_{2} \leqslant 1 / 4$. По предложению 2.2 и свойству (B2)

$$
\begin{aligned}
& H\left(x_{2}, y_{2}\right)-H\left(x_{1}, y_{2}\right)=S\left[T_{y_{2}}^{1 / 4}[g]\right]\left(x_{2}\right)-S\left[T_{y_{2}}^{1 / 4}[g]\right]\left(x_{1}\right) \\
& \quad=S\left[T_{y_{2}-y_{1}}^{1 / 4}\left[S\left[T_{y_{1}}^{1 / 4}[g]\right]\right]\right]\left(x_{2}\right)-S\left[T_{y_{2}-y_{1}}^{1 / 4}\left[S\left[T_{y_{1}}^{1 / 4}[g]\right]\right]\right]\left(x_{1}\right) \\
& \quad=S\left[T_{y_{2}-y_{1}}^{1 / 4}\left[H\left(\cdot, y_{1}\right)\right]\right]\left(x_{2}\right)-S\left[T_{y_{2}-y_{1}}^{1 / 4}\left[H\left(\cdot, y_{1}\right)\right]\right]\left(x_{1}\right) \\
& \quad \geqslant T_{y_{2}-y_{1}}^{1 / 4}\left[H\left(\cdot, y_{1}\right)\right]\left(x_{2}\right)-T_{y_{2}-y_{1}}^{1 / 4}\left[H\left(\cdot, y_{1}\right)\right]\left(x_{1}\right) \\
& \quad=H\left(x_{2}, y_{1}\right)-H\left(x_{1}, y_{1}\right)-\left(x_{2}-x_{1}\right)\left(y_{2}-y_{1}\right) .
\end{aligned}
$$

Учитывая оценку (2.8), имеем

$$
0 \geqslant H\left(x_{1}, y_{1}\right)+H\left(x_{2}, y_{2}\right)-H\left(x_{2}, y_{1}\right)-H\left(x_{1}, y_{2}\right) \geqslant-\left(x_{2}-x_{1}\right)\left(y_{2}-y_{1}\right) .
$$

Подставляя $y_{2}=1 / 4$ в (2.9), получаем

$$
0 \leqslant H\left(x_{2}, y_{1}\right)-H\left(x_{1}, y_{1}\right) \leqslant\left(1 / 4-y_{1}\right)\left(x_{2}-x_{1}\right) \leqslant \frac{1}{4}\left(x_{2}-x_{1}\right),
$$

откуда $\operatorname{Lip}_{1}(H) \leqslant 1 / 4$. Аналогичным образом убеждаемся, что $\operatorname{Lip}_{2}(H) \leqslant 1 / 4$. Продолжим $H(x, y)$ на $\mathbf{R}^{2}$, полагая

$$
H(x, y)=H(-x, y)=H(x,-y)=H(-x,-y), \quad(x, y) \in(-\infty, 0] \times[0, \infty) .
$$


Таким образом определенная функция $H$, очевидно, обладает свойством (А1), а свойство (А2) вытекает из (2.9). Оценим $\|H\|_{1}$ сверху. Имеем

$$
\begin{aligned}
\int_{-\infty}^{0} H(x, y) d x & =\int_{-\frac{k_{y} a}{16}}^{0} H(x, y) d x \leqslant \int_{-\frac{k_{y} a}{16}}^{0}\left(g(x)-g\left(-k_{y} a / 16\right)\right) d x \\
& \leqslant \sum_{k=1}^{k_{y}} \frac{a k}{16} d_{k}=\frac{a^{2} k_{y}}{1024 \ln N} .
\end{aligned}
$$

Интегрируя последнее соотношение по $y \in(0,1 / 4]$, получаем оценку

$$
\begin{aligned}
\int_{0}^{\frac{1}{4}} \int_{-\infty}^{0} H(x, y) d x d y & \leqslant \int_{0}^{(4 N \ln N)^{-1}} \frac{a^{2} N}{1024 \ln N} d y+\int_{(4 N \ln N)^{-1}}^{\frac{1}{4}} \frac{a^{2}(4 y \ln N)^{-1}}{1024 \ln N} d y \\
& \leqslant \frac{a^{2}}{4096 \ln ^{2} N}+\frac{a^{2} \ln (4 N \ln N)}{4096 \ln ^{2} N} \leqslant C_{6} \frac{a^{2}}{\ln N} \leqslant C_{6} f(a),
\end{aligned}
$$

где $0<C_{6} \leqslant 1 / 4$. Следовательно,

$$
\begin{aligned}
\|H\|_{1} & =4 \int_{0}^{\frac{1}{4}} \int_{-\infty}^{0} H(x, y) d x d y \\
& \leqslant 4 C_{6} f(a) \leqslant 4 C_{6} f\left(\left(64\|H\|_{\infty}\right) \wedge 1\right) \leqslant 4 C_{6}(64)^{3} f\left(\|H\|_{\infty}\right) .
\end{aligned}
$$

При этом $\|H\|_{1} \leqslant 4 C_{6} f(a)=4 C_{6} r \leqslant r$. Лемма 2.2 доказана.

Д о к а з а т е ль с т в о л е м м ы 2.3. Пусть $F_{X}(t)=F_{Y}(t)=(t+1 / 2)_{+} \wedge 1$, $t \in \mathbf{R}$. Условие (2.2), очевидно, выполнено. Положим $F_{X, Y}(x, y)=H(x, y)+$ $F_{X}(x) F_{Y}(y), x, y \in \mathbf{R}$. Заметим, что $F_{X, Y}$ является функцией распределения некоторого случайного вектора $(X, Y)$. Действительно, в силу (А2) приращение $F_{X, Y}$ на любом прямоугольнике неотрицательно. Легко видеть, что $F_{X, Y}$ обладает и другими свойствами функции распределения. Кроме того, $\operatorname{cov}(\mathbf{I}\{X>x\}, \mathbf{I}\{Y>y\})=$ $F_{X, Y}(x, y)-F_{X}(x) F_{Y}(y) \geqslant 0$ при всех $x, y \in \mathbf{R}$, откуда вытекает положительная ассоциированность $(X, Y)$ (см., например, [1, теорема 1.1.5]). Лемма 2.3 доказана.

3. Приложения. Рассмотрим $X=\left\{X_{t}, t \in \mathbf{R}^{d}\right\}$ - квадратично интегрируемое измеримое строго стационарное случайное поле с непрерывной ковариационной функцией. Предположим, что $X_{0}$ имеет ограниченную плотность. Пусть $W_{n}-$ последовательность растущих в смысле Ван Хова (см., например, [1, определение 3.1.1]) ограниченных измеримых множеств из $\mathbf{R}^{d}$. Возьмем произвольное $u \in \mathbf{R}$ и введем экскурсионное множество $A_{n}(u)=\left\{t \in W_{n}: X_{t}>u\right\}$. Определим $V_{n}(u)=\left|A_{n}(u)\right|$, где $|A|$ - мера Лебега множества $A \subset \mathbf{R}^{d}$. В [10] доказано, что если случайное поле $X$ квази-ассоциировано (см., например, [1, определение 1.5.8]), и

$$
\operatorname{cov}\left(X_{0}, X_{t}\right)=O\left(\|t\|_{2}^{-\alpha}\right), \quad\|t\|_{2} \rightarrow \infty,
$$

где $\alpha>3 d$, то при любом действительном $u$ последовательность случайных величин $V_{n}(u)$ удовлетворяет центральной предельной теореме (ЦПТ):

$$
\frac{V_{n}(u)-\mathbf{E} V_{n}(u)}{\sqrt{\left|W_{n}\right|}} \stackrel{d}{\rightarrow} \mathscr{N}\left(0, \sigma^{2}(u)\right), \quad n \rightarrow \infty,
$$

где

$$
\sigma^{2}(u)=\int_{\mathbf{R}^{d}} \operatorname{cov}\left(\mathbf{I}\left\{X_{0}>u\right\}, \mathbf{I}\left\{X_{t}>u\right\}\right) d t .
$$

Если наложить на $X$ более жесткое условие ассоциированности и воспользоваться следствием 1.1, то можно получить ЦПТ (3.2) при менее строгих ограничениях на ковариационную функцию $X$. Доказательство следующего результата повторяет доказательство из [10] с заменой оценки (1.2) на (1.4) и ЦПТ для $(B L, \theta)$-зависимых случайных полей на ЦПТ для ассоциированных случайных полей ([2, теорема 2$] ;$ см. также [11]). 
Теорема 3.1. Если $X \in \mathrm{A} u(3.1)$ выполнено при некотором $\alpha>2 d$, то имеет место (3.2).

Непосредственным применением ЦПТ для ассоциированных случайных полей и оценки (1.4) можно получить ЦПТ для эмпирических функций распределения. Пусть $X=\left\{X_{n}\right\}_{n \in \mathbf{Z}}$ - строго стационарная последовательность случайных величин, имеющих непрерывную ф.p. $F$. Введем случайные процессы $\beta_{n}(u)$, где $u \in \mathbf{R}$ и $n \in \mathbf{N}$, с помощью формулы

$$
\beta_{n}(u)=\sqrt{n}\left(\frac{1}{n} \sum_{k=1}^{n} \mathbf{I}\left\{X_{k} \leqslant u\right\}-F(u)\right) .
$$

Для таких процессов верна следующая теорема.

Теорема 3.2. Если $X \in \mathrm{PA}$ или $X \in \mathrm{NA}$, и для некоторого $\delta<1 / 2$

$$
\sum_{k \in \mathbf{Z}}\left|\operatorname{cov}\left(F\left(X_{0}\right), F\left(X_{k}\right)\right)\right|^{\delta}<\infty
$$

то конечномерные распределения $\beta_{n}$ сходятся $\kappa$ конечномерным распределениям чентрированного гауссовского прочесса $\beta$ с ковариачионной функцией

$$
\operatorname{cov}(\beta(u), \beta(v))=\sum_{k \in \mathbf{Z}} \operatorname{cov}\left(\mathbf{I}\left\{X_{0} \leqslant u\right\}, \mathbf{I}\left\{X_{k} \leqslant v\right\}\right), \quad u, v \in \mathbf{R} .
$$

В [4] сходимость конечномерных распределений $\beta_{n}$ установлена в предположении, что неравенство (3.3) выполнено при $\delta=1 / 3$. Заметим также, что в [12] функциональная ЦПТ для эмпирических функций распределения получена при условии $\operatorname{cov}\left(F\left(X_{0}\right), F\left(X_{k}\right)\right)=O\left(k^{-\alpha}\right), k \rightarrow \infty$, для некоторого $\alpha>4$.

Автор признателен профессору А. В. Булинскому за постановку задачи и внимание к работе. Автор также благодарен рецензенту за ценные замечания, способствовавшие улучшению изложения.

\section{СПИСОК ЛИТЕРАТУРЫ}

1. Булинский А.В., Шашкин А. П. Предельные теоремы для ассоциированных случайных полей и родственных систем. М.: Физматлит, 2008.

2. Newman C. M. Normal fluctations and the FKG inequalities. - Commun. Math. Phys., 1980, v. 74, № 2, p. 119-128.

3. Bagai I., Prakasa Rao B.L.S. Estimation of the survival function for stationary associated processes. - Statist. Probab. Lett., 1991, v. 12, p. 385-391.

4. Yu H. A Glivenko-Cantelli lemma and weak convergence for empirical processes of associated sequences. - Probab. Theor. Related Fileds, 1993, v. 95, № 3, p. 357-370.

5. Matula P., Ziemba M. General covariance inequalities. — Cent. Eur. J. Math., 2011, v. 9, № 2 (2011), p. 281-293.

6. Matula $P$. A note on some inequalities for certain classes of positively dependent random variables. - Probab. Math. Statist., 2004, v. 24, № 1, p. 17-26.

7. Esary J.D., Proschan F., Walkup D.W. Association of random variables, with applications. - Ann. Math. Statist., 1967, v. 38, № 5, p. 1466-1474.

8. Newman C.M. Asymptotic independence and limit theorems for positively and negatively dependent random variables. Inequalities in Statistics and Probability. Hayward, CA, Institute of Mathematical Statistics, 1984, p. 127-140.

9. Joag-Dev K., Proschan F. Negative association of random variables, with applications. - Ann. Statist., 1983, v. 11, № 1, p. 286-295.

10. Bulinski A. V., Spodarev E., Timmermann F. Central limit theorems for the excursion set volumes of weakly dependent random fields. - Bernoulli, 2012, v. 18, № 1, p. 100118.

11. Булинский A. В. Центральная предельная теорема для положительно ассоциированных стационарных случайных полей. - Вестн. С.-Петерб. ун-та., 2011, сер. 1, вып. 2, с. 5-13. 
12. Louhichi $S$. Weak convergence for empirical processes of associated sequences. - Ann. Inst. Henri Poincare, Probabilites et Statistiques, 2000, v. 36, №, p. 547-567.

Поступила в редакцию 24.X.2012

(C) 2013 г.

FEINBERG E.A.* KASYANOV P.O.**, ZADOIANCHUK N. V.**

\title{
FATOU'S LEMMA FOR WEAKLY CONVERGING PROBABILITIES ${ }^{1)}$
}

\begin{abstract}
Лемма Фату утверждает при определенных условиях, что интеграл нижнего предела последовательности функций не превышает нижнего предела соответствующих интегралов. Данная заметка описывает подобные неравенства, когда, в отличие от случая одной меры, функции интегрируются относительно разных мер, которые образуют слабо сходящуюся последовательность.
\end{abstract}

Ключевые слова и фразы: лемма Фату, вероятность, мера, слабая сходимость.

1. The inequality for nonnegative functions. Consider a measurable space $(S, \mathscr{B})$, where $S$ is a metric space and $\mathscr{B}$ is its Borel $\sigma$-field. Let $\mathbf{P}(S)$ be the set of probability measures on $(S, \mathscr{B}(S))$. According to Fatou's lemma, [8], for any $\mu \in \mathbf{P}(S)$ and for any sequence of nonnegative measurable functions $f_{1}, f_{2}, \ldots$

$$
\int_{S} \liminf _{n \rightarrow+\infty} f_{n}(s) \mu(d s) \leqslant \liminf _{n \rightarrow+\infty} \int_{S} f_{n}(s) \mu(d s) .
$$

A sequence of probability measures $\left\{\mu_{n}\right\}_{n \geqslant 1}$ from $\mathbf{P}(S)$ converges weakly to $\mu \in \mathbf{P}(S)$ if for any bounded continuous function $f$ on $S$

$$
\int_{S} f(s) \mu_{n}(d s) \rightarrow \int_{S} f(s) \mu(d s) \quad \text { as } n \rightarrow+\infty .
$$

A sequence of probability measures $\left\{\mu_{n}\right\}$ from $\mathbf{P}(S)$ converges setwise to $\mu \in \mathbf{P}(S)$ if (1.2) holds for any bounded measurable function $f$. If $\left\{\mu_{n}\right\}_{n \geqslant 1} \subset \mathbf{P}(S)$ converges setwise to $\mu \in \mathbf{P}(S)$, according to Royden [5, p. 231], for any sequence of nonnegative measurable function $f_{1}, f_{2}, \ldots$

$$
\int_{S} \liminf _{n \rightarrow+\infty} f_{n}(s) \mu(d s) \leqslant \liminf _{n \rightarrow+\infty} \int_{S} f_{n}(s) \mu_{n}(d s) .
$$

However, this is not true, if $\mu_{1}, \mu_{2}, \ldots$ converge weakly to $\mu$.

Indeed, let $S=[0,1], \mu_{n}(A)=\mathbf{I}\{1 / n \in A\}, \mu(A)=\mathbf{I}\{0 \in A\}$ for $A \in \mathscr{B}([0,1])$, and $f(s)=f_{n}(s)=\mathbf{I}\{s=0\}$ for $n=1,2, \ldots$ and $s \in[0,1]$. Then $\int_{S} f(s) \mu(d s)=1$, $\int_{S} f(s) \mu_{n}(d s)=0$, and (1.3) does not hold.

Theorem 1.1 presents Fautou's lemma for weakly converging measures $\mu_{n}$ and nonnegative functions $f_{n}$. This fact is useful for the analysis of Markov decision processes

* Department of Applied Mathematics and Statistics, Stony Brook University, Stony Brook, NY 11794-3600, USA; e-mail: eugene.feinberg@sunysb.edu

** Institute for Applied System Analysis, National Technical University of Ukraine «Kyiv Polytechnic Institute», Peremogy ave., 37, Build. 35, 03056, Kyiv, Ukraine; e-mail: kasyanov@i.ua; ninellll@i.ua

1) This research was partially supported by NSF grants CMMI-0928490 and CMMI1335296, by the Ukrainian State Fund for Fundamental Research under grant GP/F49/070, and by grant 2273/13 from the National Academy of Sciences of Ukraine. 